\title{
Star clusters: age, metallicity and extinction from integrated spectra
}

\author{
Rosa M. González Delgado ${ }^{1}$ and Roberto Cid Fernandes ${ }^{2}$ \\ ${ }^{1}$ Instituto de Astrofísica de Andalucía-CSIC, Granada, Spain \\ ${ }^{2}$ Departamento de Física, Universidade Federal de Santa Catarina, Florianopolis, Brazil
}

\begin{abstract}
Integrated optical spectra of star clusters in the Magellanic Clouds and a few Galactic globular clusters are fitted using high-resolution spectral models for single stellar populations. The goal is to estimate the age, metallicity and extinction of the clusters, and evaluate the degeneracies among these parameters. Several sets of evolutionary models that were computed with recent high-spectral-resolution stellar libraries (MILES, GRANADA, STELIB), are used as inputs to the STARLIGHT code to perform the fits. The comparison of the results derived from this method and previous estimates available in the literature allow us to evaluate the pros and cons of each set of models to determine star cluster properties. In addition, we quantify the uncertainties associated with the age, metallicity and extinction determinations resulting from variance in the ingredients for the analysis.
\end{abstract}

Keywords. galaxies: star clusters, galaxies: stellar content, Magellanic Clouds

\section{Introduction}

Star clusters (SCs) are one of the main modes of star formation in galaxies. They are powerful tracers of the formation and evolution history of galaxies because they retain information about the parent galaxy. In this respect, the age, metallicity and mass of cluster populations are key information to test different galaxy-formation scenarios.

Evolutionary synthesis models are powerful tools to infer the properties of extragalactic clusters, since their integrated light is easily modelled by a single-age and singlemetallicity stellar population (SSP). For this reason, they are very useful objects to check the capabilities and uncertainties of the stellar population models.

In this contribution, we estimate the age, metallicity and extinction of a sample of clusters in the Magellanic Clouds (Large and Small: LMC and SMC) and Milky Way by fitting their optical spectra. We use several sets of high-resolution spectral stellar population models to quantify the accuracies and uncertainties associated with the age, extinction and metallicity determinations of stellar clusters that results from variance in the model ingredients.

\section{The sample and data}

The sample analysed here consists of 27 SCs from Leonardi \& Rose (2003; LR03), 20 of which belong to the LMC, three to SMC and four are Galactic globular clusters (GCs). The spectra cover $3650-4600 \AA$, with a final resolution of $5.7 \AA$.

With respect to the literature data, we have compiled the ages and metallicities derived by LR03 for the MC clusters, and for the GC the values obtained by Koleva et al. (2008). LR03 adopted ages based on CMDs and/or derived from a secondary indicator, the $S$ parameter, based on the $U-B$ and $B-V$ integrated colors (calibrated 
by Girardi et al. 1995). For the clusters' metallicities, we have taken the values from Olszewski et al. (1991), obtained by measuring the CaII triplet in individual stars in the SCs.

\section{Set of evolutionary models and method}

The stellar library is one of the most important ingredients in evolutionary synthesis models. In the last few years, several groups have spent a lot of effort to build complete stellar libraries at high/intermediate spectral resolution at optical wavelengths. As a result, several sets of models are currently available for spectral synthesis (see, e.g., the review by González Delgado 2009). Here, we have used seven versions of the most up-todate high-resolution spectral models. They are:

- Galaxev and STELIB: the models (Bruzual \& Charlot 2003, BC03) are computed with the STELIB library (Le Borgne et al. 2003), Salpeter (BC94s, BC00s) and Chabrier (BC94c, BC00c) initial mass functions (IMFs), and the evolutionary tracks from the Padova group, (1994 isochrones: BC94s, BC94c; 2000 isochrones: BC00s, BC00c). STELIB contains 249 stellar spectra in the 3200-9500 $\AA$ range at a resolution of $3 \AA$.

- SED@ and GRANADA: the GRANADA stellar library (Martins et al. 2005) contains 1654 synthetic spectra with a sampling of $0.3 \AA$, covering the range $3000-7000$ $\AA$, for a wide range of effective temperatures (3000-55000 K), gravity ( -0.5 to 5.0$)$, and metallicity (solar, half and 0.2 solar). The library was implemented in the SED@ code (Cerviño \& Luridiana 2006) to calculate SSP models (González Delgado et al. 2005) using the Padova 2000 isochrones and a Salpeter IMF (RG00s).

- Vazdekis and MILES: the MILES library (Sánchez-Blázquez et al. 2006) contains a thousand stars spanning a large range of stellar parameters covering the $3500-7500 \AA$ range at a resolution of $2.3 \AA$. The library was incorporated into the code by Vazdekis et al. (2009). They compute SSP spectra with the Padova 2000 isochrones and a Salpeter IMF (V00s).

- Galaxev and MILES/GRANADA: these models are analogous to those in BC03. The STELIB library was replaced by a combination of the MILES and GRANADA stellar libraries (Charlot \& Bruzual, in prep.) (CB94c).

To determine the stellar population properties for each cluster, we fit the entire observed spectrum using the STARLIGHT code (Cid Fernandes et al. 2005) and the set of models described above. The version of STARLIGHT used here provides the following results:

- The best fit: for each of the SSP model suites, the best SSP is obtained as that characterized by the minimum $\chi^{2}$.

- Multi-population fit: the code combines several SSPs and provides for each set of a given metallicity a population vector that represents the fractional contribution of each SSP of a given age to the model flux at a given wavelength.

- Bayesian estimates: following the Bayes theorem, the posterior probability for age, metallicity and extinction is given for each set of models.

\section{Results and conclusions}

Table 1 presents the Bayesian age, metallicity and extinction for all clusters. Figures 1 and 2 show some of the results obtained. A more extensive discussion of the quality of the fits and a comparison with literature data is included in Cid Fernandes \& González Delgado (2009) and González Delgado \& Cid Fernandes (2009). 
Table 1. The Bayesian age, metallicity and extinction for all clusters obtained with the models by Charlot \& Bruzual (2009; CB94c: columns 2-4), González Delgado et al. (2005; RG00s: columns 6-7), Vazdekis et al. (2009; V00s: columns 8-10)

\begin{tabular}{|c|c|c|c|c|c|c|c|c|c|}
\hline $\mathrm{Name}$ & $\log (\mathrm{age} / \mathrm{yr})$ & $\log Z / Z \odot$ & $A_{V}$ & $\log (\operatorname{age} / \mathrm{yr})$ & $\log Z / Z_{\odot}$ & $A_{V}$ & $\log ($ age $/$ yr $)$ & $\log Z / Z \odot$ & $A_{V}$ \\
\hline M 411 & $9.26 \pm 0.00$ & $-0.68 \pm 0.02$ & $0.17 \pm 0.04$ & $9.13 \pm 0.07$ & $-0.52 \pm 0.17$ & $0.06 \pm 0.06$ & $9.13 \pm 0.10$ & $-0.68 \pm 0.20$ & $0.41 \pm 0.11$ \\
\hline M 416 & $9.53 \pm 0.07$ & $-0.71 \pm 0.17$ & $0.02 \pm 0.08$ & $9.28 \pm 0.02$ & $-0.38 \pm 0.00$ & $0.01 \pm 0.01$ & $9.90 \pm 0.01$ & $-1.28 \pm 0.00$ & $0.22 \pm 0.02$ \\
\hline M 419 & $9.24 \pm 0.08$ & $-0.64 \pm 0.17$ & $0.15 \pm 0.04$ & $9.06 \pm 0.03$ & $-0.39 \pm 0.07$ & $0.09 \pm 0.04$ & $9.10 \pm 0.00$ & $-0.67 \pm 0.06$ & $0.46 \pm 0.05$ \\
\hline NG C 1651 & $9.40 \pm 0.02$ & $-0.68 \pm 0.03$ & $0.14 \pm 0.09$ & $9.24 \pm 0.04$ & $-0.50 \pm 0.15$ & $0.04 \pm 0.04$ & $9.58 \pm 0.19$ & $-1.10 \pm 0.34$ & $0.36 \pm 0.19$ \\
\hline N G C 1754 & $9.94 \pm 0.00$ & $-1.68 \pm 0.00$ & $0.29 \pm 0.05$ & $9.22 \pm 0.03$ & $-0.39 \pm 0.06$ & $0.02 \pm 0.02$ & $9.81 \pm 0.03$ & $-1.28 \pm 0.04$ & $0.02 \pm 0.02$ \\
\hline NG C 1783 & $9.11 \pm 0.17$ & $-0.29 \pm 0.35$ & $0.14 \pm 0.13$ & $9.19 \pm 0.05$ & $-0.49 \pm 0.15$ & $0.03 \pm 0.03$ & $9.25 \pm 0.06$ & $-0.69 \pm 0.13$ & $0.26 \pm 0.10$ \\
\hline NG C 1795 & $9.41 \pm 0.04$ & $-0.68 \pm 0.05$ & $0.11 \pm 0.09$ & $9.25 \pm 0.04$ & $-0.53 \pm 0.15$ & $0.05 \pm 0.05$ & $9.66 \pm 0.16$ & $-1.21 \pm 0.25$ & $0.38 \pm 0.17$ \\
\hline N G C 1806 & $9.33 \pm 0.01$ & $-0.68 \pm 0.00$ & $0.42 \pm 0.04$ & $9.25 \pm 0.02$ & $-0.65 \pm 0.08$ & $0.10 \pm 0.05$ & $9.26 \pm 0.02$ & $-0.67 \pm 0.03$ & $0.34 \pm 0.08$ \\
\hline NG C 1818 & $7.41 \pm 0.07$ & $+0.35 \pm 0.16$ & $0.48 \pm 0.19$ & $7.39 \pm 0.02$ & $-0.38 \pm 0.03$ & $0.45 \pm 0.05$ & $8.00 \pm 0.00$ & $-1.68 \pm 0.00$ & $0.02 \pm 0.02$ \\
\hline N G C 1831 & $8.61 \pm 0.07$ & $-0.38 \pm 0.01$ & $0.15 \pm 0.14$ & $8.75 \pm 0.01$ & $-0.68 \pm 0.00$ & $0.03 \pm 0.03$ & $8.73 \pm 0.03$ & $-0.40 \pm 0.08$ & $0.03 \pm 0.04$ \\
\hline N G C 1846 & $9.31 \pm 0.00$ & $-0.68 \pm 0.01$ & $0.25 \pm 0.07$ & $9.24 \pm 0.03$ & $-0.65 \pm 0.08$ & $0.04 \pm 0.04$ & $9.23 \pm 0.07$ & $-0.70 \pm 0.13$ & $0.27 \pm 0.12$ \\
\hline N G C 1866 & $8.01 \pm 0.00$ & $+0.42 \pm 0.03$ & $0.56 \pm 0.04$ & $8.20 \pm 0.00$ & $0.00 \pm 0.00$ & $0.29 \pm 0.03$ & $8.05 \pm 0.03$ & $+0.19 \pm 0.07$ & $0.53 \pm 0.07$ \\
\hline N G C 1978 & $9.54 \pm 0.02$ & $-0.68 \pm 0.00$ & $0.15 \pm 0.04$ & $9.29 \pm 0.02$ & $-0.38 \pm 0.02$ & $0.03 \pm 0.03$ & $9.42 \pm 0.11$ & $-0.69 \pm 0.10$ & $0.11 \pm 0.09$ \\
\hline NG C 2010 & $8.05 \pm 0.03$ & $+0.41 \pm 0.07$ & $0.13 \pm 0.08$ & $8.20 \pm 0.01$ & $0.00 \pm 0.00$ & $0.03 \pm 0.03$ & $8.14 \pm 0.04$ & $+0.14 \pm 0.09$ & $0.09 \pm 0.07$ \\
\hline N G C 2121 & $9.57 \pm 0.06$ & $-0.68 \pm 0.01$ & $0.17 \pm 0.10$ & $9.33 \pm 0.05$ & $-0.46 \pm 0.14$ & $0.11 \pm 0.09$ & $9.99 \pm 0.19$ & $-1.23 \pm 0.17$ & $0.55 \pm 0.19$ \\
\hline NG C 2133 & $8.38 \pm 0.03$ & $+0.02 \pm 0.10$ & $0.19 \pm 0.11$ & $8.35 \pm 0.01$ & $0.00 \pm 0.02$ & $0.26 \pm 0.05$ & $8.27 \pm 0.03$ & $+0.11 \pm 0.10$ & $0.33 \pm 0.09$ \\
\hline NG C 2134 & $8.87 \pm 0.22$ & $-1.94 \pm 0.81$ & $0.06 \pm 0.07$ & $8.39 \pm 0.07$ & $-0.24 \pm 0.18$ & $0.14 \pm 0.11$ & $8.41 \pm 0.08$ & $-0.29 \pm 0.19$ & $0.09 \pm 0.10$ \\
\hline N G C 2136 & $7.90 \pm 0.03$ & $+0.41 \pm 0.07$ & $0.60 \pm 0.08$ & $7.95 \pm 0.05$ & $-0.41 \pm 0.10$ & $0.62 \pm 0.10$ & $8.12 \pm 0.11$ & $-0.47 \pm 0.19$ & $0.40 \pm 0.19$ \\
\hline NG C 2203 & $9.39 \pm 0.02$ & $-0.67 \pm 0.04$ & $0.42 \pm 0.09$ & $9.27 \pm 0.04$ & $-0.55 \pm 0.15$ & $0.12 \pm 0.07$ & $9.35 \pm 0.05$ & $-0.69 \pm 0.09$ & $0.25 \pm 0.10$ \\
\hline N G C 2210 & $9.86 \pm 0.09$ & $-1.69 \pm 0.09$ & $0.20 \pm 0.05$ & $9.20 \pm 0.02$ & $-0.41 \pm 0.10$ & $0.02 \pm 0.02$ & $9.80 \pm 0.00$ & $-1.68 \pm 0.00$ & $0.10 \pm 0.03$ \\
\hline N G C 2213 & $9.37 \pm 0.02$ & $-0.68 \pm 0.02$ & $0.32 \pm 0.06$ & $9.27 \pm 0.03$ & $-0.65 \pm 0.08$ & $0.04 \pm 0.03$ & $9.30 \pm 0.00$ & $-0.68 \pm 0.01$ & $0.13 \pm 0.05$ \\
\hline N G C 2214 & $7.85 \pm 0.01$ & $+0.42 \pm 0.00$ & $0.15 \pm 0.04$ & $7.90 \pm 0.01$ & $-0.38 \pm 0.04$ & $0.14 \pm 0.03$ & $8.04 \pm 0.01$ & $-0.38 \pm 0.01$ & $0.02 \pm 0.02$ \\
\hline N G C 2249 & $8.96 \pm 0.00$ & $-0.68 \pm 0.01$ & $0.01 \pm 0.020$ & $8.79 \pm 0.05$ & $-0.68 \pm 0.00$ & $0.06 \pm 0.14$ & $8.84 \pm 0.02$ & $-0.67 \pm 0.04$ & $0.15 \pm 0.06$ \\
\hline $47 \mathrm{Tuc}$ & $9.98 \pm 0.03$ & $-0.37 \pm 0.03$ & $0.02 \pm 0.020$ & $9.90 \pm 0.08$ & $-0.60 \pm 0.13$ & $0.06 \pm 0.06$ & $9.98 \pm 0.05$ & $-0.37 \pm 0.04$ & $0.02 \pm 0.02$ \\
\hline M 15 & $9.85 \pm 0.00$ & $-1.68 \pm 0.01$ & $0.05 \pm 0.030$ & $9.14 \pm 0.27$ & $-0.41 \pm 0.14$ & $0.06 \pm 0.20$ & $9.80 \pm 0.00$ & $-1.68 \pm 0.00$ & $0.02 \pm 0.02$ \\
\hline M 79 & $9.93 \pm 0.02$ & $-1.68 \pm 0.00$ & $0.01 \pm 0.010$ & $9.21 \pm 0.03$ & $-0.43 \pm 0.11$ & $0.02 \pm 0.02$ & $9.84 \pm 0.11$ & $-1.68 \pm 0.00$ & $0.01 \pm 0.01$ \\
\hline NG C 1851 & $9.61 \pm 0.05$ & $-0.68 \pm 0.00$ & $0.01 \pm 0.010$ & $9.31 \pm 0.02$ & $-0.38 \pm 0.01$ & $0.02 \pm 0.02$ & $10.2 \pm 0.04$ & $-1.28 \pm 0.00$ & $0.11 \pm 0.04$ \\
\hline
\end{tabular}

Our main conclusions are:

- All models can be used to derive ages that are in good agreement both with each other and with literature data, but they are slightly older than those based on the $S-$ CMD method (see Figure 1).

- There is less agreement between the models for metallicity and extinction. In particular, Galaxev/STELIB models underestimate the metallicity by 0.6 dex (see Figure 2), while the extinction is overestimated by $0.1 \mathrm{mag}$.

- The age-metallicity relation for LMC clusters is well reproduced by the spectral fitting method, except for Galaxev/STELIB models.

- Accuracies of about 0.1 dex in age and 0.3 dex in metallicity can be achieved with the GRANADA and MILES libraries as long as the models are not extrapolated beyond their expected range of validity.

\section{Acknowledgements}

We acknowledge support from a joint $\mathrm{CNPq}-\mathrm{CSIC}$ bilateral collaboration grant, and from grant AYA2007-64712.

\section{References}

Bruzual, G. \& Charlot, S. 2003, MNRAS, 344, 1000

Cerviño, M. \& Luridiana, V. 2006, A\&A, 413, 145

Cid Fernandes, R., Mateus, A., Sodré, L., Stasińska, G., \& Gomes, J. M. 2005, MNRAS, 358, 363

Cid Fernandes, R. \& González Delgado, R. M. 2009, MNRAS, submitted

Girardi, L., Chiosi, C., Bertelli, G., \& Bressan, A. 1995, A\&A A, 298, 87

González Delgado, R. M., Cerviño, M., Martins, L. P., Leitherer, C., \& Hauschildt, P. H. 2005, MNRAS, 357, 945 

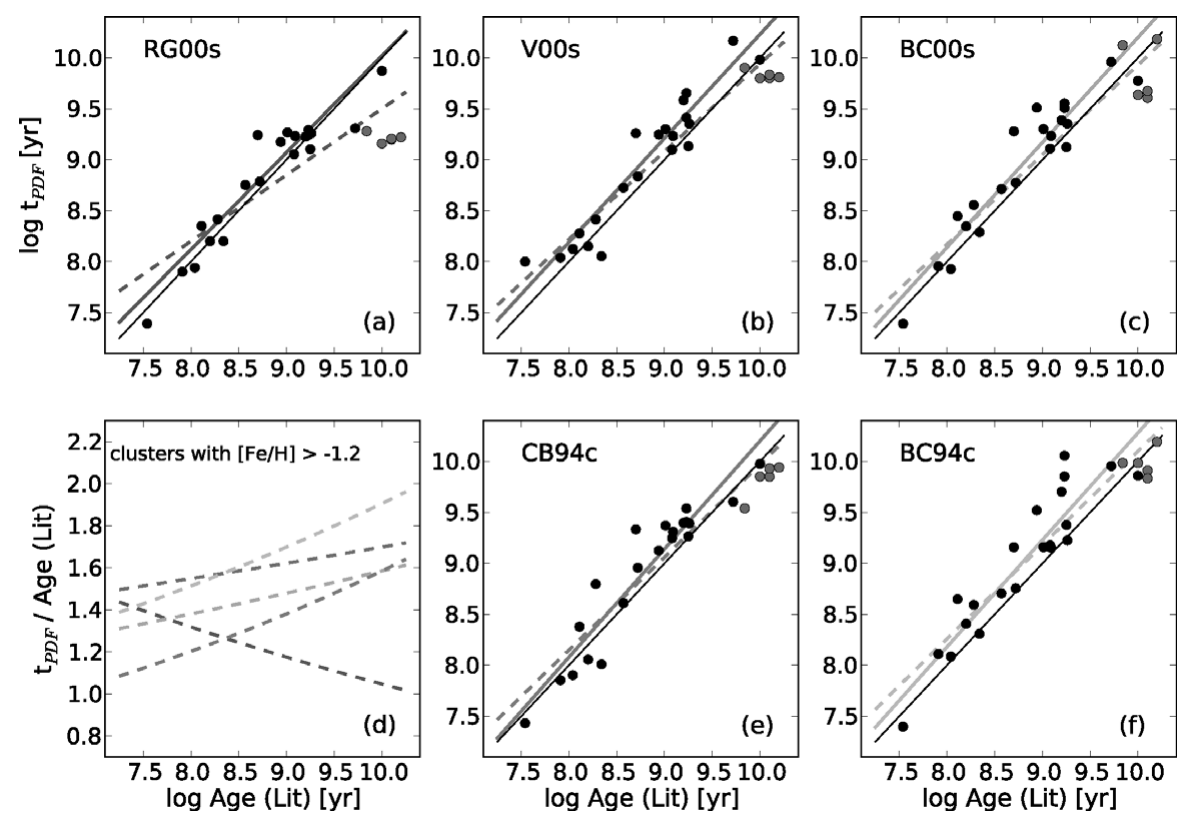

Figure 1. Panels $a, b, c, e$ and $f$ : Correlation between the age obtained with starlight and ages in the literature derived using the $S-\mathrm{CMD}$ calibration. Each panel represents the result for each set of models. Metal-poor clusters $\left(\log \left(Z / \mathrm{Z}_{\odot}\right) \leqslant-1.2\right)$ are marked with magenta circles. The black line is the one-to-one relationship, while the dashed line is a linear fit to all points and the solid line is a linear fit excluding the metal-poor clusters. The bottom left-hand panel shows the deviation of the linear fit obtained for each set of models with respect to the one-to-one relationship, expressed as the ratio between the spectral fit and the literature ages.
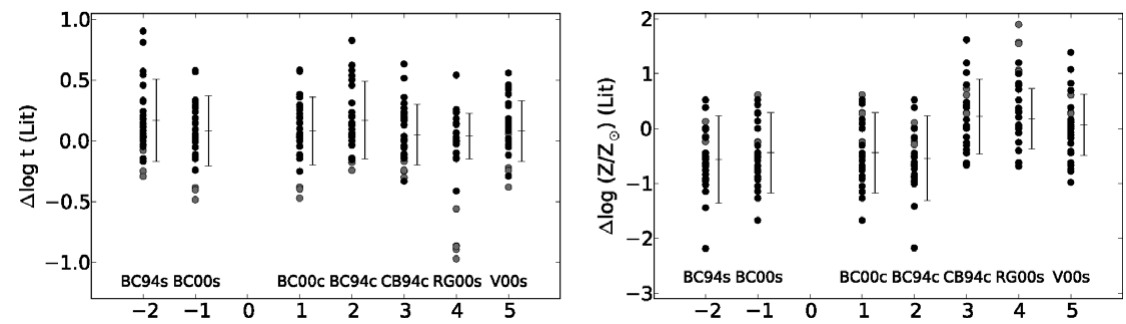

Figure 2. Logarithmic difference between the spectral-fit age (left) or metallicity (right) obtained for each set of models and the literature values. Magenta circles mark the metal-poor clusters $\left(\log \left(Z / \mathrm{Z}_{\odot}\right) \leqslant-1.2\right)$. Error bars mark the mean and standard deviation for each set of models.

González Delgado, R. M. 2009, in: E. Pérez, R. de Grijs \& R. M. González-Delgado (eds.), Young massive star clusters: initial conditions and environments, ApSS, 324, 231

González Delgado, R. M. \& Cid Fernandes, R. 2009, MNRAS, submitted

Le Borgne J. -F., Bruzual, G., Pelló, R., Lançon, A., Rocca-Volmerange, B., Sanahuja, B.,

Schaerer, D., Soubiran, C., \& Vílchez-Gómez, R. 2003, A\&A, 402, 433

Leonardi, A. J. \& Rose J. A. 2003, ApJ, 126, 1811

Koleva, M., Prugniel, P., Ocvirk, P., Le Borgne, D., \& Soubiran, C. 2008, MNRAS, 385, 1998

Martins, L. P., González Delgado, R. M., Leitherer, C., Cerviño, M., \& Hauschildt, P. H. 2005, MNRAS, 358, 49

Olszewski, E. W., Schommer, R. A., Suntzeff, N. B., \& Harris, H. C. 1991, AJ, 101, 515

Vazdekis, A., et al. 2009, MNRAS, submitted 